\section{Tales from the crypt}

\author{
By Lev Osherovich, Senior Writer
}

Separate studies by Dutch and U.S. researchers have identified a subset of cells that drive tumor growth in colon cancer. ${ }^{1,2}$ These cancer stem cells could potentially be targeted to wipe out colorectal tumors at the source, which is an area of the intestinal epithelium called the intestinal crypt. The discoveries in one of the papers have been licensed by Agamyxis BV, a new biotech cofounded by the study's author.

The crypts are pocket-like structures hidden within the folds of the intestinal epithelium. They produce a steady stream of epithelial cells that push their way to the intestinal surface. The two papers, both published in Nature, build on earlier efforts to identify molecular markers of the endlessly dividing stem cells at the base of the crypts.

In the new studies, independent teams at the Hubrecht Institute for Developmental Biology and Stem Cell Research and St.
"When we delete Apc in stem cells, we get explosive tumors, but if we delete it in other cells, the mice are fine." -Hans Clevers, Agamyxis BV when Apc was knocked out. This suggests that Lgr5-positive stem cells, not their descendants, are the bona fide source of tumors.

The St. Jude team reached similar conclusions, though by a different route. That team examined a surface protein called prominin 1 (Prom1; CD133) in a different mouse model of colorectal cancer.

CD133 has been found in normal stem cells in a variety of other tissues and is thought to mark cells as susceptible to transformation, said team leader Richard Gilbertson, an associate member in the Department of Developmental Neurobiology and Oncology at St. Jude.

"We've been interested in comparing cancer stem cells with regular stem cells," he added. "Because there are stem cell-like cells in cancer, it's tempting to speculate that they arise directly from normal cells with proliferative capacity."

Gilbertson's team found that CD133, like Lgr5, was expressed only in cells at the base of the intestinal crypt. The team stained the crypt cells with an inducible yellow fluorescent protein and watched the stain move through the crypt over time, finding that all of the cells of the intestinal epithelium started life as CD133-positive crypt cells.

To test whether CD133-positive cells played a role in colorectal tumors, Gilbertson's team engineered a mouse strain with a hyperactive form of $\beta$-catenin, a transcription factor that drives tumor growth. $\beta$-catenin expression in CD133positive cells led to tumor formation. In contrast, Jude Children's Research Hospital used mouse genetics to examine how cells at the bottom of the crypt affect the growth of colorectal adenomas.

\section{Cryptic commands}

The Dutch study was led by Hans Clevers, director of the Hubrecht Institute, and examined the role of a poorly understood protein called leucine-rich repeat-containing $G$ protein-coupled receptor 5 (LGR5; GPR49) in a mouse model of colon cancer.

In 2007, Clevers' team reported that Lgr5 was expressed in mouse crypt stem cells, but not in cells further up the sides of the crypt, and that cells lost the ability to divide indefinitely when they stopped expressing Lgr $5 .^{3}$ Because colon cancer cells also divide indefinitely, the team hypothesized that Lgr5 could be a marker of the most proliferative cells in colorectal tumors.

To test this idea, the team disrupted the adenomatous polyposis coli $(A p c)$ gene in cells with or without Lgr5. Apc is a tumor suppressor that puts the brakes on cell division in the colon and is absent in many colorectal cancers.

The team found that removal of Apc in Lgr5-positive cells indeed led to tumor formation, whereas knocking out $A p c$ in neighboring cells had no effect.

"When we delete $A p c$ in stem cells, we get explosive tumors, but if we delete it in other cells, the mice are fine," Clevers told SciBX.

Moreover, cells that had already migrated out of the base of the crypt, and thus had stopped making Lgr5, did not develop into tumors cells lacking CD133 did not respond to $\beta$-catenin expression.

\section{Marker or target?}

Both studies go a long way toward proving the cancer stem cell hypothesis, which predicts that a small subset of cells within a tumor is the real culprit behind the cancer. Most cancer therapeutics kill tumor cells with incomplete efficiency and may thus let a small minority of hidden cancer stem cells survive; identifying the true origin of a tumor is a major goal for drug development.

The problem is that targeting cells that bear LGR5 and CD133 is a challenge, since healthy versions of these cells are likely to be essential for the normal workings of the intestinal crypt.

As a result, Gilbertson believes that neither protein is likely to be a good direct drug target. "Both CD133 and LGR5 are expressed both in tumors and in the normal stem cells, so if you target them the result could be horrible," he said.

Indeed, targeting all CD133- or LGR5-positive cells could kill healthy stem cells and prevent the intestinal lining from undergoing its daily process of self-renewal.

Nevertheless, said Gilbertson, "you can now look for drugs that selectively hit the malignant population" of tumor cells expressing CD133 and LGR5.

However, Clevers thinks that LGR5 is a "potentially excellent colon cancer target," noting that although only about $2 \%$ of the cells in actively growing tumors are LGR5 positive, eradicating this subpopulation of cells would kill off the source of the remaining cancer cells. 


\section{TARGETS \& MECHANISMS}

Instead, Clevers sees the markers as an opportunity to identify cancer stem cells and screen those cells for "another marker that is really responsible" for tumor growth. To this end, Gilbertson plans to isolate CD133-expressing tumor cells from the intestine and compare their pattern of gene expression with normal CD133-positive cells.

Clevers and the Royal Netherlands Academy of Arts and Sciences have sought patents on the use of LGR5 and a related protein, LGR6, as cancer stem cell markers and potential targets in regenerative medicine. The patents are being licensed to Agamyxis, a cancer company cofounded by Clevers, who is also the company's CSO.

Clevers and Agamyxis CEO Ton Logtenberg cofounded vaccine company Crucell N.V.

Agamyxis is focused on small molecule inhibitors of NOTCH1, an enzyme of the $\gamma$-secretase family that is a target in Barrett's disease, intestinal adenoma and adenocarcinoma. The company did not disclose whether the findings in Clevers' study relate to its $\mathrm{NOTCH}$ program.

According to Clevers, Agamyxis' lead compound is in preclinical development.
Gilbertson has not patented his team's findings.

Osherovich, L. SciBX 2(4); doi:10.1038/scibx.2009.125

Published online Jan. 29, 2009

\section{REFERENCES}

1. Barker, N. et al. Nature published online Dec. 17, 2008; doi:10.1038/nature07602

Contact: Hans Clevers, Hubrecht Institute for Developmental Biology and Stem Cell Research, Utrecht, the Netherlands e-mail: h.clevers@niob.knaw.nl

2. Zhu, L. et al. Nature published online Dec. 17, 2008;

doi:10.1038/nature07589

Contact: Richard Gilbertson, St. Jude Children's Research Hospital, Memphis, Tenn.

e-mail: richard.gilbertson@stjude.org

3. Barker, N. et al. Nature 449, 1003-1007 (2007)

COMPANIES AND INSTITUTIONS MENTIONED

Agamyxis BV, Utrecht, the Netherlands

Crucell N.V. (Euronext:CRXL; NASDAQ:CRXL), Leiden, the Netherlands Hubrecht Institute for Developmental Biology and Stem Cell

Research, Utrecht, the Netherlands

Royal Netherlands Academy of Arts and Sciences, Amsterdam, the Netherlands

St. Jude Children's Research Hospital, Memphis, Tenn. 